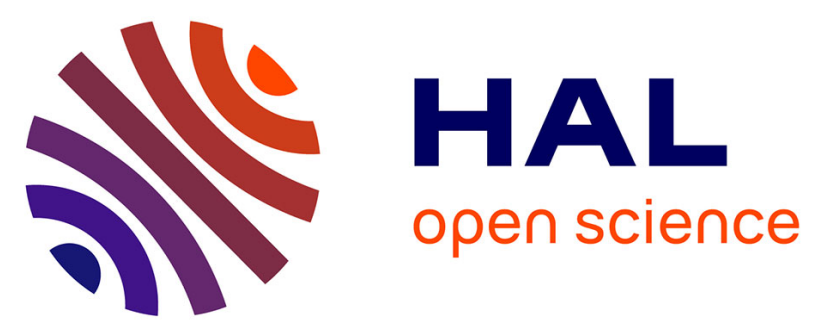

\title{
AlN film thickness effect on photoluminescence properties of AlN/carbon nanotubes shell/core nanostructures for deep ultra-violet optoelectronic devices
}

N. Ouldhamadouche, A. Achour, K. Ait. Aissa, M. Islam, A. Ahmadpourian, A. Arman, M. A. Soussou, M. Chaker, L. Le Brizoual, M. A. Djouadi

\section{To cite this version:}

N. Ouldhamadouche, A. Achour, K. Ait. Aissa, M. Islam, A. Ahmadpourian, et al.. AlN film thickness effect on photoluminescence properties of AlN/carbon nanotubes shell/core nanostructures for deep ultra-violet optoelectronic devices. Thin Solid Films, 2017, 622, pp.23-28. 10.1016/j.tsf.2016.12.018 . hal-01502346

HAL Id: hal-01502346

https://hal-univ-rennes1.archives-ouvertes.fr/hal-01502346

Submitted on 19 Jun 2017

HAL is a multi-disciplinary open access archive for the deposit and dissemination of scientific research documents, whether they are published or not. The documents may come from teaching and research institutions in France or abroad, or from public or private research centers.
L'archive ouverte pluridisciplinaire HAL, est destinée au dépôt et à la diffusion de documents scientifiques de niveau recherche, publiés ou non, émanant des établissements d'enseignement et de recherche français ou étrangers, des laboratoires publics ou privés. 


\section{AIN Film Thickness Effect on Photoluminescence}

\section{Properties of AIN/Carbon Nanotubes Shell/Core}

\section{Nanostructures for Deep Ultra-Violet Optoelectronic}

\section{Devices}

N.Ouldhamadouche ${ }^{1, *}$, A. Achour ${ }^{2, *}$, K. Ait. Aissa ${ }^{3}$, M. Islam ${ }^{4}$, A. Ahmadpourian ${ }^{5}$, A.

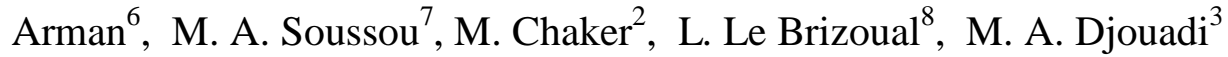

${ }^{1}$ Laboratoire de génie physique Université Ibn Khaldoun, BP P 78 zaâroura 14000 Tiaret, Algeria

${ }^{3}$ Institut National de la Recherche Scientifique (INRS), 1650 Boulevard Lionel-Boulet, Varennes, QC J3X 1P7, Canada.

${ }^{3}$ Université de Nantes, CNRS, Institut des Matériaux Jean Rouxel, UMR 6502, 2 rue de la Houssinière B.P. 32229 - 44322 Nantes cedex 3, France.

${ }^{4}$ Deanship of Scientific Research, Advanced Manufacturing Institute, King Saud University, P.O. Box 800, Riyadh, 11421, Saudi Arabia

${ }^{5}$ Department of Physics, Kermanshah Branch, Islamic Azad University, Kermanshah, Iran

${ }^{6}$ Young Researchers and Elite Club, Kermanshah Branch, Islamic Azad University, Kermanshah, Iran

${ }^{7}$ Research \& Technology Center of Energy, Thermal Processes Laboratory, Hammam Lif, 2050 Tunis, Tunisia 
${ }^{8}$ Institut d'Electronique et de Télécommunications, IETR - UMR CNRS 6164, Campus de Beaulieu -Bat 11D 263 Av General Leclerc 35042, Rennes cedex, France.

\footnotetext{
* These authors contributed equally to this work
}

To whom correspondence should be addressed: a_aminph@yahoo.fr

\section{ABSTRACT}

Aluminum nitride (AIN) nanostructures are very attractive in various optoelectronic applications such as deep ultraviolet light emitting devices. The fabrication of these AlN nanostructures with good crystalline quality and compatibility in line with other microfabrication processes has significant importance for practical applications. AlN films of different thickness values were deposited via DC reactive magnetron sputtering over vertically aligned multiwalled carbon nanotube (CNTs) arrays to obtain AlN/CNTs verticallyaligned shell/core nanostructure assembly. Such hybrid nanostructures were characterized using scanning electron microscope, transmission electron microscope, X-ray diffraction, Raman spectroscopy and time-resolved photoluminescence spectroscope (TR-PL) techniques. The results indicated that AlN/CNTs have a nanorods structure morphology with good AlN crystalline quality. The PL measurements revealed a maximum increase in the luminescent intensity of the exciton band in case of AlN/CNTs with $600 \mathrm{~nm}$ thick AlN layer, which is 
many orders of magnitude higher than that of AlN film produced over silicon substrate. It is anticipated that synergistic effects of CNTs and AlN through an increase in the specific surface area and oxygen-induced defects cause enhancement in the photoluminescence properties, making these hybrid nanostructures a promising candidate for optoelectronic applications.

Keywords: Aluminum nitride, carbon nanotubes, reactive magnetron sputtering, hybrid nanostructures, Photoluminescence

\section{Introduction}

Aluminium nitride $(\mathrm{AlN})$ is a material with remarkable properties including large optical band gap value $\left(\mathrm{E}_{\mathrm{g}}\right.$ 5.9-6.2eV), high hardness and good thermal conductivity [1]. It can be used in several electronic and optoelectronic devices, ultraviolet detector, light emitting diodes and thermal interface materials, to name a few $[2,3]$. The large $E_{g}$ value for AlN permits its incorporation into deep UV LEDs [4-6] and extreme UV detectors [7], the devices that can be employed for probing intrinsic florescence in protein, equipment/personnel decontamination, photocatalysis and astronomy $[1,8]$. In order to obtain AIN with efficient UV emission, a fabrication process that will produce AlN with high structural quality, is desired. The UV emission of AlN can be enhanced by increasing its surface area, that in turn, may be possible in case of AlN nanostructures with morphologies as diverse as nanowires, nanotubes, nanorods, nanotips, hierarchical comb-like structures, pine like structures or nanobelts [9]. In most cases, however, the synthesis techniques carry disadvantages of high processing 
temperature $\left(800-1200{ }^{\circ} \mathrm{C}\right)[10,11]$ and poor structural quality of the resulting AlN films with deterioration in band gap emission besides emission in the visible spectral region [12-14].

In this work, vertically aligned carbon nanotubes (CNTs) were grown over silicon substrates followed by sputter deposition of AlN films for any enhancement in the surface area and photoluminescence properties. Similar strategy was adopted by our group in case of zinc oxide $(\mathrm{ZnO})$ [15] and titanium nitride (TiN) films produced over CNTs arrays [16]. Herein, a fabrication route compatible with micro-fabrication techniques was developed via dc reactive magnetron sputtering (dcMS) of AlN films over vertically-aligned CNTs arrays to obtain AlN/CNT shell/core nanostructures at low temperature. In addition, the ability to grow vertically aligned CNTs at relatively low temperature of $420^{\circ} \mathrm{C}$ [17] offers the advantage of low cost processing.

In contrast with AlN films over polished silicon substrate, the AlN/CNT hybrid nanostructures exhibited band gap emission intensity of much larger magnitude in the deep UV spectral region with relatively little contribution from defect emission. Such shell/core hybrid nanostructures offer strong potential for used in deep UV light emitting devices and other optoelectronic applications.

\section{Experimental details}

\subsection{Plasma enhanced chemical vapour deposition growth of vertically aligned CNT arrays}

Vertically aligned carbon nanotubes (CNTs) arrays were grown over silicon substrates using distributed electron-cyclotron-resonance (ECR) plasma enhanced chemical vapor deposition (PECVD) process. The details of the experimental setup are described elsewhere [18]. A 10 
$\mathrm{nm}$ thick nickel (Ni) film was obtained via DC-plasma sputter deposition and then transferred to the PECVD reactor. Upon heating to $600{ }^{\circ} \mathrm{C}$, the film transformed into Ni nanoparticles, $\sim 50 \mathrm{~nm}$ in diameter, that subsequently catalyzed CNT growth in a PECVD reactor using ECR plasma and acetylene to ammonia $\mathrm{C}_{2} \mathrm{H}_{2}: \mathrm{NH}_{3}$ ratio of 1:4, other parameters being chamber pressure of $0.2 \mathrm{~Pa}, 125 \mathrm{~W}$ microwave power and synthesis time of $1 \mathrm{~h}$.

\subsection{DC plasma reactive sputtering of AIN films}

Over both $\mathrm{Si}(100)$ substrates and CNTs arrays grown over $\mathrm{Si}(100)$, AlN films were deposited through DC reactive magnetron sputtering technique using aluminum target $(50 \mathrm{~mm}$ in diameter, $99.999 \%$ purity), Argon/ $\mathrm{N}_{2}$ gas mixture atmosphere and a target-substrate distance of $30 \mathrm{~mm}$. An unbalanced magnetron sputtering system powered by a $150 \mathrm{~W}$ DC power supply was used. Before deposition, the target was cleaned using argon gas discharge plasma for 15 min followed by a pre-sputtering step with a shutter shielding the sample under the same conditions as those during subsequent film deposition. Once residual pressure of $<6 \times 10^{-}$

${ }^{6} \mathrm{~Pa}$ was achieved, the chamber pressure was increased to $0.4 \mathrm{~Pa}$ and was kept at that level during AlN deposition. The total gas flow rate during deposition was maintained constant at $40 \mathrm{sccm}$ with $\mathrm{N}_{2}$ flow rate of $14 \mathrm{sccm}$. The substrates remained at a floating potential and were neither additionally heated nor cooled during the deposition. In order to check the effect of the thickness on the photoluminescence (PL) signal, different samples were prepared by varying the deposited AlN film thickness from 50 to $1500 \mathrm{~nm}$.

\subsection{Characterizations}

The scanning electron microscope (SEM) studies were performed for morphology and thickness of the deposited hybrid nanostructures using a JEOL JSM 7600 apparatus. The images were recorded at an accelerating voltage of $5 \mathrm{kV}$. The morphology was also 
investigated using field-emission gun scanning electron microscope (FEG-SEM) (JEOL; JSM7600F1) and field-emission gun transmission electron microscope (FEG-TEM; Hitachi HF2000) operating at $200 \mathrm{kV}$. For TEM observation, the samples were micro-delaminated with a diamond tip and directly transferred onto the TEM copper grids. The AlN/CNTs hybrid structures were also characterized using X-ray diffraction (XRD) apparatus (D500 MOXTEK) with monochromatic $\mathrm{CuK} \alpha$ radiation $(\lambda=0.154 \mathrm{~nm})$ in the $\theta-2 \theta$ Bragg Brentano configuration. The room temperature Raman spectra were recorded with a Jobin Yvon micro Raman spectrometer (T64000) at $240 \mathrm{~nm}$ wavelength and $100 \mathrm{~mW}$ power. The time-resolved PL measurements were performed on a system consisting of a monochromator $(1.3 \mathrm{~m})$ and a frequency quadrupled 100 fs Ti:Saphire laser with excitation photon energy set at about 6.28 eV. A single photon counting detection system together with a micro-channel-plate photomultiplier tube (with 20 ps time resolution) or a streak camera ( 2 ps time resolution) was used to record time-resolved spectra.

\section{Results and discussion}

The AlN films with thickness values in the range of 50 to $1500 \mathrm{~nm}$ thick were produced over the CNT arrays, as shown in surface and cross-section SEM view of the AlN/CNT hybrid structures in Fig. 1. The cross-section view revealed vertically-aligned shell/core configuration of the AlN/CNT nanorod assembly with varying degrees of porosity and AlN film thickness values from 50 to $1500 \mathrm{~nm}$ (Fig1. b, d, f, h). Due to the line-of-sight deposition, the aligned CNT arrays undergo encapsulation of the individual nanotubes with AlN film at and near their ends, thus resulting in a convex shape whose size increases with an increase in the thickness of the deposited layer. While the surface microstructures, presented in Fig. 1(a, c, e, g) indicate the AlN/CNT grain size as well as the film density at the surface or near-surface area, the cross-section view reveals the degree of CNT encapsulation and 
filling of the inter-nanotubes spacing by the incoming flux of reactively-sputtered AlN. Upon increasing the deposition time, the grain size, as estimated from the surface microstructure, increases from $\sim 100 \mathrm{~nm}$ to $\sim 1 \mu \mathrm{m}$ with an associated decrease in the degree of surface porosity. Examination of the cross-section morphology indicated an increase in the degree of CNT encapsulation, both axially as well as radially, for longer deposition times as manifested by AlN deposition over greater length segments of the CNT forest and larger size of the AIN/CNT hybrid structure at and near the top of CNT array. Since the average length of the core/shell vertically-aligned nanostructure array $(3.4 \mu \mathrm{m})$ is slightly higher than that for pure CNT array $(2.2 \mu \mathrm{m})$ in case of $1500 \mathrm{~nm}$ thick AlN film, it is deduced that (i) AlN deposition does not completely fill the spacing between nanotubes in the aligned CNT array and (ii) beyond a certain deposition time, the top surface of the CNT array is completely buried underneath AlN thus resulting in a AlN-rich top layer. From SEM studies, it seems that AlN films with thickness values of 50, 400 and $600 \mathrm{~nm}$ maintain high surface area of the AlN/CNT hybrid structure. For these thickness values, the AlN deposition apparently induced an inverse pyramid- or conical shaped columnar morphology with a decrease in the degree of CNT encapsulation from its upper end to the base. The AlN attachment primarily to the upper end of the aligned nanotubes forest may result in higher luminescence intensity of band gap emission in such AlN/CNT shell/core nanostructures. In the case of $1500 \mathrm{~nm}$ thick AlN film, a continuous, compact layer albeit with less surface area is, likely to have formed over the top of CNT array. All these aspects will probably affect the band gap emission of AlN deposit.

The XRD patterns of the AlN films produced over vertically-aligned CNT arrays are presented in Fig. 2. The hybrid structures exhibit two different crystallographic orientations indexed as $c$-axis (002) and (101) planes, both of which may be associated with hexagonal Wurtzite structure of AlN (JCPDS: No. 08-0262) [19]. It was noticed that upon increasing the 
AlN film thickness from 400 to $1500 \mathrm{~nm}$, while the intensity of the (101) diffraction peak remains almost unchanged, there is a progressive increase in the peak intensity corresponding to the (002) plane.

One of the AlN@CNT core/shell hybrid nanostructure sample with AlN film thickness of 600 nm was examined under TEM, as shown in Fig. 3. From low magnification image of the region near the tip of AlN/CNT array (Fig. 3a), complete encapsulation of the nanotubes is noticed. Although, a cursory look at the HRTEM micorstructure of the tip area suggests layered petal-like morphology, a closer look reveals rodlike-morphology in the form of bundles (Fig. 3b). The dense, columnar morphology with c-axis (002)-preferred crystallographic orientation, produced at low deposition pressure $(0.3 \mathrm{~Pa})$ and $\sim 35 \% \mathrm{~N}_{2}$ gas concentration, is promoted by an increased in the mobility of adatoms and is in agreement with earlier studies on reactively sputtered AlN films $[20,21]$. The selected area electron diffraction pattern (SAED) of a relatively large area of an individual AlN@CNT shell/core structure (Fig. 3c) exhibits diffraction rings characteristic of the (101) and (002) crystallographic orientations of the AlN lattice. The presence of the sharp, diffraction spots in a circular arrangement points towards polycrystalline nature of AlN with relatively large grains [22]. The SAED of a smaller region confirms (Fig. 3d) hexagonal structure of the AIN coating with a (002) preferential orientation.

Fig.4 shows the UV Raman spectrum in the range $200-1200 \mathrm{~cm}^{-1}$ for AlN/CNT shell/core hybrid nanostructures with different AlN film thickness values. For $50 \mathrm{~nm}$ thick film, no spectroscopy mode was observed. All the three Raman active modes namely $A_{1}, E_{1}$ and $E_{2}$, are noticed to be present in the Raman spectra for AlN/CNT samples with AlN film thickness of 400, 600 and $1500 \mathrm{~nm}$. Both $A_{1}$ and $E_{1}$ are polar modes with each of them having longitudinal (LO) and transverse (TO) modes arising from the interaction of the vibrations with the long-range Coulomb field [23]. From the Raman spectra, five (5) different peaks 
positioned at frequencies of 248,610,657, 669 and $910 \mathrm{~cm}^{-1}$ are seen. The weak phonon peak at $248 \mathrm{~cm}^{-1}$ corresponds to the $\mathrm{E}_{2}$ (low) (also denoted as $\mathrm{E}_{2}{ }^{\mathrm{L}}$ ) mode, whereas the peak at 657 $\mathrm{cm}^{-1}$ represents the $\mathrm{E}_{2}$ (high) (also called $\mathrm{E}_{2}{ }^{\mathrm{H}}$ ) mode. Similarly, while the phonon mode $\mathrm{A}_{1}(\mathrm{TO})$ can be clearly identified at $610 \mathrm{~cm}^{-1}$, the $\mathrm{A}_{1}(\mathrm{LO})$ mode maybe present at $890 \mathrm{~cm}^{-1}$ as a shoulder peak for the $910 \mathrm{~cm}^{-1}$ peak. The peaks located at 669 and $910 \mathrm{~cm}^{-1}$ correspond to the $\mathrm{E}_{1}(\mathrm{TO})$ and $\mathrm{E}_{1}(\mathrm{LO})$ phonon modes, respectively [23, 24]. Predominance of the active Raman modes depends on the direction of phonon propagation, whether parallel or perpendicular to the c-axis of the crystal [25]. Such discussion is beyond the scope of this research and the Raman studies presented herein confirm stoichiometric AlN film formation from dc reactive sputtering.

AlN has a relatively small $c / a$ ratio of 1.601 and a band gap energy value of $6.113 \mathrm{eV}$ including free exciton binding energy of $80 \mathrm{meV}$ [26]. In bulk AlN ceramics, the excitation wavelength of $195 \mathrm{~nm}$ causes appearance of different bands in the emission spectrum at spectral positions of $300-500 \mathrm{~nm}, 600 \mathrm{~nm}, 700 \mathrm{~nm}$ and $1000 \mathrm{~nm}$ with respective characteristic features of a broad UV-blue band (300-500 nm), a narrower band and minor bands at 700 and $1000 \mathrm{~nm}$. The broad complex UV-blue band is composed of two subbands at 400 and $480 \mathrm{~nm}$, each of which has its own excitation spectrum. While the $400 \mathrm{~nm}$ emission band causes appearance of excitation band centred at $240-250 \mathrm{~nm}$, the $480 \mathrm{~nm}$ emission band gives rise to an excitation band at 280-290 $\mathrm{nm}$ [27]. Both types of emission bands are also excited at $\sim 210$ $\mathrm{nm}(5.8-5.9 \mathrm{eV})$. The exciton generation is attributed to recombination of two defect centers, such as a complex donor defect formed via substitution of nitrogen ion $\mathrm{O}_{\mathrm{N}}$ by an oxygen ion in a regular site and a neighboring aluminum vacancy $\left.\mathrm{v}_{\mathrm{Al}}\left(\mathrm{O}_{\mathrm{N}}-\mathrm{v}_{\mathrm{Al}}\right)\right)$ and a closely situated oxygen ion (an acceptor $\mathrm{O}_{\mathrm{N}}$ ). In some cases, implantation of oxygen ions may cause damage to the AlN surface layers thus increasing oxygen concentration, and in turn, generating defect 
centers [28]. At room temperature, therefore, the presence of impurities and defects cause radiative recombination energies at $5.69,5.85$ and $6.09 \mathrm{eV}$ to represent bound exciton recombinations in AIN [29]. The room temperature photoluminescence (PL) spectra of different AIN/CNT vertically-aligned shell/core nanostructures were obtained, as presented graphically in Fig. 5. These were compared with the PL spectrum of the 1500-nm thick AlN film produced over silicon substrate (called ref. AlN). The choice for the ref. AlN sample was made owing to its high thickness value and superior structural quality. In case of ref. AlN, a weak exciton peak at $\sim 5.8 \mathrm{eV}$ is noticed, the presence of which may be attributed to the band gap emission at 400 and $480 \mathrm{~nm}$. The reasons for such low luminescent intensity maybe high structural quality of the film and low defect density at the film surface due to very small amounts of residual oxygen in the chamber during film deposition.

When AlN is reactively sputter deposited over aligned CNT array as in the case of different AlN/CNT shell/core nanostructures, an intense, broad and asymmetric peak is seen at $5.85 \mathrm{eV}$ in the PL spectra. The peak intensity increases with an increase in the AlN film thickness reaching a maximum value for $600 \mathrm{~nm}$ thick AlN film over the CNT array. While the intensity for this sample is much greater than that for $1500 \mathrm{~nm}$ thick AlN film over silicon, The PL intensity, however, drops once the AlN layer thickness over CNT is $1500 \mathrm{~nm}$. Nevertheless, it still remains more intense than the one obtained for the ref AlN film. Furthermore, the relatively sharp deep-UV emission peak is an indication of good structural quality of the AIN film deposited over vertically-aligned CNT array. The increase of the band gap emission intensity of CNT/AIN compared to the ref AlN film maybe attributed to the increase in the surface-to-volume ratio of AlN/CNT shell/core nanostructures that exhibit a nanorod-like structure with a convex end i.e. nanoscale conical shape at the top of the vertically-aligned CNT forest. In fact, the peak intensity increases with AlN thickness up to $600 \mathrm{~nm}$ and then decreases for higher thicknesses due to the decrease in the surface area (Fig. 
1). The band gap emission intensities of 50 and 400 nm thick AlN@CNT are lower than that of $600 \mathrm{~nm} \mathrm{AlN}$, even though the surface area values for the 50 and $400 \mathrm{~nm}$ thick CNT/AlN nanostructures seem to be higher (Fig. 1). This may be caused by an improvement in the crystalline quality of the AlN film upon a thickness increase from 400 to $600 \mathrm{~nm}[30,31]$. The maximization of the PL intensity upon varying AlN film thickness value maybe attributed to the combined effect of an increase in the specific surface area of the hybrid nanostructures as well as enhancement in the oxygen concentration with subsequently higher defect density.

\section{Conclusions}

The dc plasma reactive magnetron sputtering technique can be employed to obtain AlN/CNT shell/ core nanostructures over vertically aligned multiwalled carbon nanotubes. The AlN films produced at low chamber pressure and $35 \% \mathrm{~N}_{2}$ gas flow are near-stoichiometric with predominantly c-axis (002) orientation and have dense, columnar morphology comprising of bundled nanorods configuration. While an increase in the AlN film thickness from of 50 to $600 \mathrm{~nm}$ over aligned CNT array promotes photoluminescence in the exciton spectrum owing to greater specific surface area and enhanced surface defect density, the CNT array is completed embedded by the AlN film upon further increase in thickness to $1500 \mathrm{~nm}$, with subsequent deterioration in the PL properties. As compared to pure AlN film, the AlN/CNT hybrid shell/core assembly with $600 \mathrm{~nm}$ thick AlN film exhibits maximum photoluminescence intensity. The enhancement in the PL intensity of the AlN band gap emission in the deep UV regime presumably arises from the trade-off between AlN structural quality and changes in the defect density owing to any change in the specific surface area. The significant enhancement in the PL intensity of the AlN films upon interfacing with CNT 
highlights the importance and potential utility of such shell/core nanostructure assemblies in multifunctional optoelectronic devices.

\section{Acknowledgements}

The authors would like to extend their sincere appreciation to the Deanship of Scientific Research at King Saud University for its funding of this research through the Research Group Project no. RGP-283.

\section{List of Figures}

Fig.1: SEM images of the surface microstructure and cross-section of the AlN/CNT vertically aligned shell/core arrays with AlN film thickness of (a, b) $50 \mathrm{~nm},(\mathrm{c}, \mathrm{d}) 400 \mathrm{~nm},(\mathrm{e}, \mathrm{f}) 600 \mathrm{~nm}$ and $(\mathrm{g}, \mathrm{h}) 1500 \mathrm{~nm}$.

Fig.2: XRD patterns of the AlN/CNT shell/core nanostructures with different AlN film thickness values.

Fig. 3: (a) TEM micrograph of the AlN/CNT sample with 600-nm AlN film over verticallyaligned CNT array, (b) High-resolution microstructure of the hybrid assembly near tip area and (c, d) SAED patterns of large and small areas of the microstructure presented in (b).

Fig.4: Raman spectra of the AlN/CNT shell/core hybrid nanostructures with different thickness values for the AlN films. 
Fig.5: PL spectra of the AlN/CNT shell/core nanostructures with different AlN film thickness and pure AlN films produced over silicon substrate. 


\section{References}

[1] A. Sedhain, N. Nepal, M.L. Nakarmi, T.M. Al tahtamouni, J.Y. Lin, H.X. Jiang, Z. Gu,

J.H. Edgar, Photoluminescence properties of AlN homoepilayers with different orientations, Applied Physics Letters, 93 (2008) 041905.

[2] G. Radhakrishnan, Properties of AlN films grown at $350 \mathrm{~K}$ by gas-phase excimer laser photolysis, Journal of Applied Physics, 78 (1995) 6000-6005.

[3] C. Duquenne, M.P. Besland, P.Y. Tessier, E. Gautron, Y. Scudeller, D. Averty, Thermal conductivity of aluminium nitride thin films prepared by reactive magnetron sputtering, Journal of Physics D: Applied Physics, 45 (2012) 015301.

[4] Y. Taniyasu, M. Kasu, N. Kobayashi, Intentional control of n-type conduction for Sidoped AlN and $\mathrm{Al}_{\mathrm{XGa}} \mathrm{1}_{-\mathrm{XN}}(0.42 \leq \mathrm{x}<1)$, Applied Physics Letters, 81 (2002) 1255-1257. [5] Y. Taniyasu, M. Kasu, T. Makimoto, Electrical conduction properties of n-type Si-doped AlN with high electron mobility (> $\left.100 \mathrm{~cm}^{2} \mathrm{~V}^{-1} \mathrm{~s}^{-1}\right)$, Applied Physics Letters, 85 (2004) 4672 4674.

[6] Y. Taniyasu, M. Kasu, T. Makimoto, Increased electron mobility in n-type Si-doped AlN by reducing dislocation density, Applied Physics Letters, 89 (2006) 182112.

[7] H. Hirayama, Quaternary InAlGaN-based high-efficiency ultraviolet light-emitting diodes, Journal of Applied Physics, 97 (2005) 091101.

[8] A. Khan, K. Balakrishnan, T. Katona, Ultraviolet light-emitting diodes based on group three nitrides, Nat Photon, 2 (2008) 77-84.

[9] W. Lei, D. Liu, J. Zhang, B. Liu, P. Zhu, T. Cui, Q. Cui, G. Zou, AlN nanostructures: tunable architectures and optical properties, Chemical Communications, (2009) 1365-1367. [10] S.-C. Shi, S. Chattopadhyay, C.-F. Chen, K.-H. Chen, L.-C. Chen, Structural evolution of AlN nano-structures: Nanotips and nanorods, Chemical Physics Letters, 418 (2006) 152-157. 
[11] L. Trinkler, B. Berzina, D. Kasjan, L.C. Chen, Luminescence properties of AlN nanostructures revealed under UV light irradiation, Journal of Physics: Conference Series, 93 (2007) 012040.

[12] S.L. Yang, R.S. Gao, P.L. Niu, Z.Y. Zou, R.H. Yu, Arc Discharge Synthesis and Photoluminescence of 3D Feather-like AIN Nanostructures, Nanoscale Res Lett, 6 (2011) 12. [13] X.H. Ji, Q.Y. Zhang, S.P. Lau, H.X. Jiang, J.Y. Lin, Temperature-dependent photoluminescence and electron field emission properties of AlN nanotip arrays, Applied Physics Letters, 94 (2009) 173106.

[14] Y. Tamura, K. Hane, AlN Nanowall Structures Grown on Si (111) Substrate by Molecular Beam Epitaxy, Nanoscale Research Letters, 10 (2015) 1-5.

[15] N. Ouldhamadouche, A. Achour, I. Musa, K. Ait Aissa, F. Massuyeau, P.Y. Jouan, M. Kechouane, L. Le Brizoual, E. Faulques, N. Barreau, M.A. Djouadi, Structural and photoluminescence characterization of vertically aligned multiwalled carbon nanotubes coated with ZnO by magnetron sputtering, Thin Solid Films, 520 (2012) 4816-4819. [16] A. Achour, J.B. Ducros, R.L. Porto, M. Boujtita, E. Gautron, L. Le Brizoual, M.A. Djouadi, T. Brousse, Hierarchical nanocomposite electrodes based on titanium nitride and carbon nanotubes for micro-supercapacitors, Nano Energy, 7 (2014) 104-113.

[17] A. Achour, A.A. El Mel, N. Bouts, E. Gautron, E. Grigore, B. Angleraud, L. Le Brizoual, P.Y. Tessier, M.A. Djouadi, Carbon nanotube growth at $420{ }^{\circ} \mathrm{C}$ using nickel/carbon composite thin films as catalyst supports, Diamond and Related Materials, 34 (2013) 76-83. [18] D. Blazei, Thermal inteface materials, Electronic Cooling, 9 (2003) 14-20.

[19] L.S. Chuah, Z. Hassan, H. Abu Hassan, Influence of Al monolayers on the properties of AlN layers on Si (111), Surface Review and Letters, 16 (2009) 99-103. 
[20] H. Cheng, Y. Sun, J.X. Zhang, Y.B. Zhang, S. Yuan, P. Hing, AlN films deposited under various nitrogen concentrations by RF reactive sputtering, Journal of Crystal Growth, 254 (2003) 46-54.

[21] M. Ishihara, S.J. Li, H. Yumoto, K. Akashi, Y. Ide, Control of preferential orientation of AlN films prepared by the reactive sputtering method, Thin Solid Films, 316 (1998) 152-157. [22] S. Khamseh, M. Nose, T. Kawabata, A. Saiki, K. Matsuda, K. Terayama, S. Ikeno, Effect of Deposition Conditions on the Structure and Properties of CrAlN Films Prepared by Pulsed DC Reactive Sputtering in FTS Mode at High Al Content, MATERIALS TRANSACTIONS, 49 (2008) 2082-2090.

[23] H. Hiroshi, Properties of GaN and related compounds studied by means of Raman scattering, Journal of Physics: Condensed Matter, 14 (2002) R967.

[24] V.Y. Davydov, Y.E. Kitaev, I.N. Goncharuk, A.N. Smirnov, J. Graul, O. Semchinova, D. Uffmann, M.B. Smirnov, A.P. Mirgorodsky, R.A. Evarestov, Phonon dispersion and Raman scattering in hexagonal GaN and AlN, Physical Review B, 58 (1998) 12899-12907.

[25] L.E. McNeil, M. Grimsditch, R.H. French, Vibrational Spectroscopy of Aluminum Nitride, Journal of the American Ceramic Society, 76 (1993) 1132-1136.

[26] J. Li, K. B. Nam, M. L. Nakarmi, J. Li, J. Y. Lin, H. X. Jiang, P. Carrier, S. H. Wei, Band structure and fundamental optical transitions in wurtzite AlN, Applied Physics Letters, 83 (2003) 5163.

[27] B. Berzina, L. Trinkler, D. Jakimovica, V. Korsaks, J. Grabis, I. Steins, E. Palcevskis, S. Belluci, L. Chen, L.-Ch. ; S. Chattopadyay, K.-H. Chen, Spectral charcterisation of bulk and nanostructured aluminum nitride. Journal of Nanophotonics, 3 (2009) 1-16.

[28] L. Trinkler, B. Berzina, A. Auzina, M. Benabdesselam, P. Iacconi, UV light energy storage and thermoluminescence in AlN ceramics, Phys. Stat. Sol., 4(3) (2007) 1032-1035. 
[29] X. Tang, F. R. B. Hossain, K. Wongchotigul, M. G. Spencer, Mater. Res. Soc. Symp. Proc. 449 (1997) 119

[30] K. Ait Aissa, A. Achour, J. Camus, L. Le Brizoual, P.Y. Jouan, M.A. Djouadi, Comparison of the structural properties and residual stress of AlN films deposited by dc magnetron sputtering and high power impulse magnetron sputtering at different working pressures, Thin Solid Films, 550 (2014) 264-267.

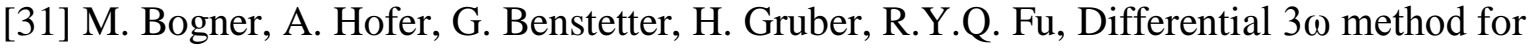
measuring thermal conductivity of $\mathrm{AlN}$ and $\mathrm{Si}_{3} \mathrm{~N}_{4}$ thin films, Thin Solid Films, 591, Part B (2015) 267-270. 



Fig. 1 


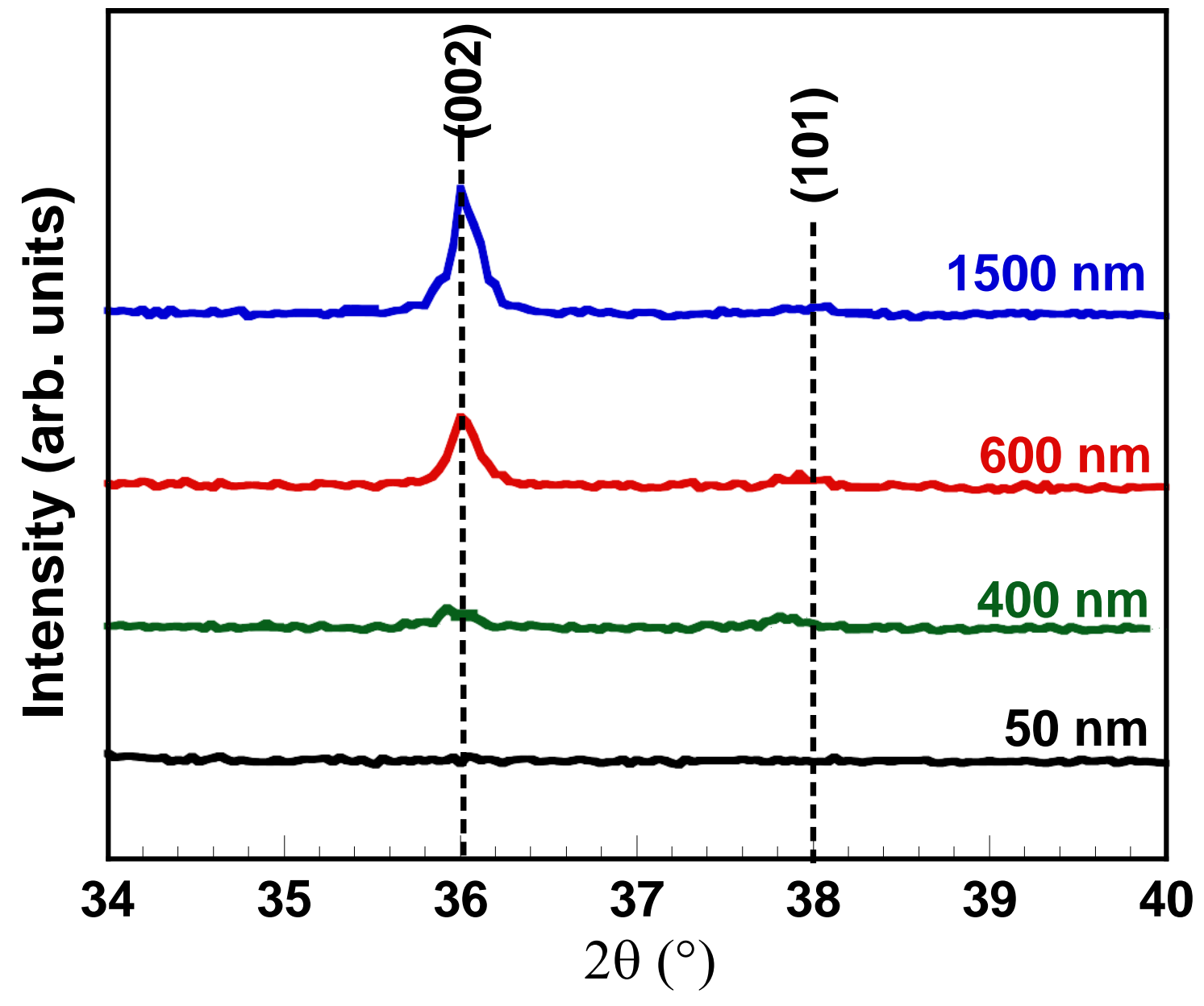

Fig. 2 


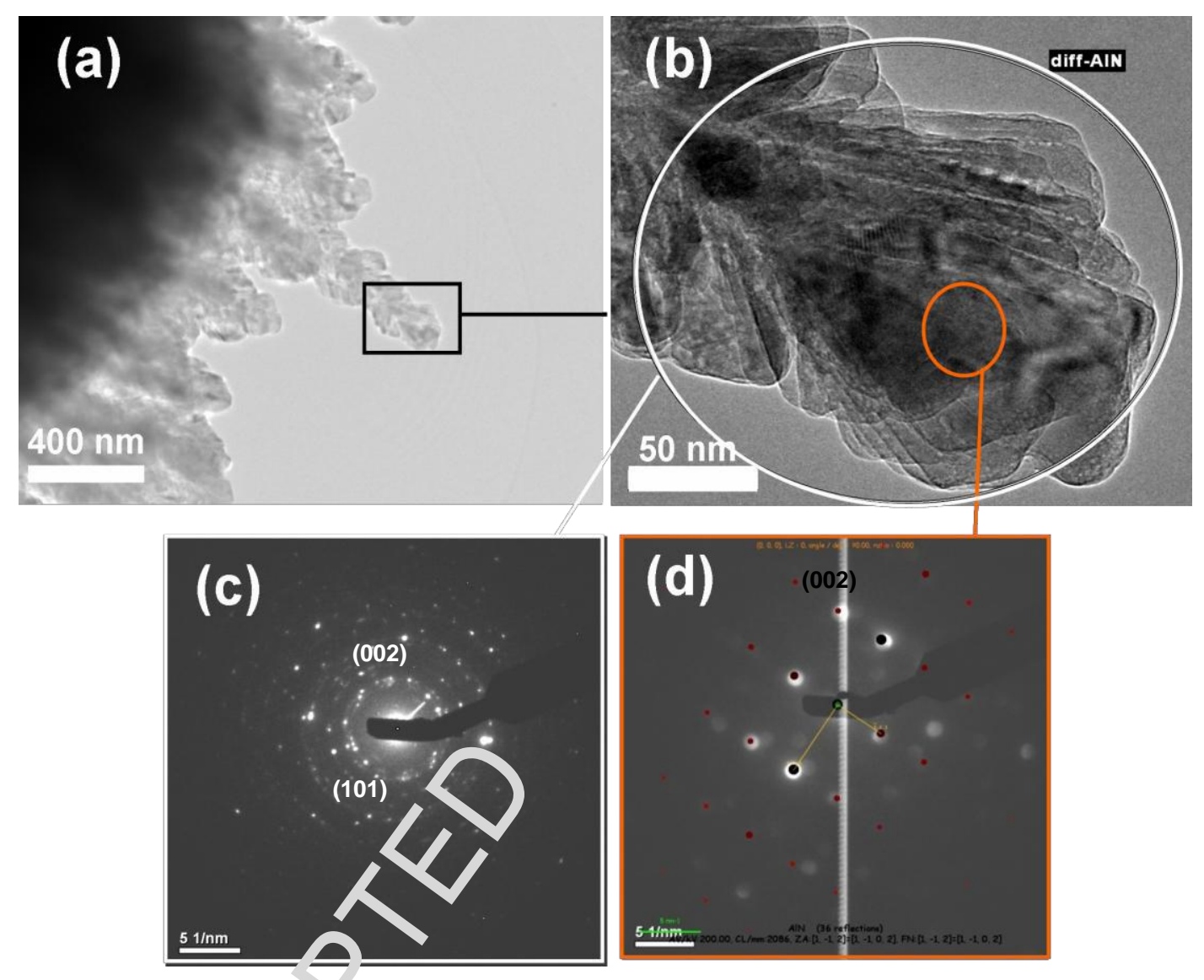

Fig. 3 


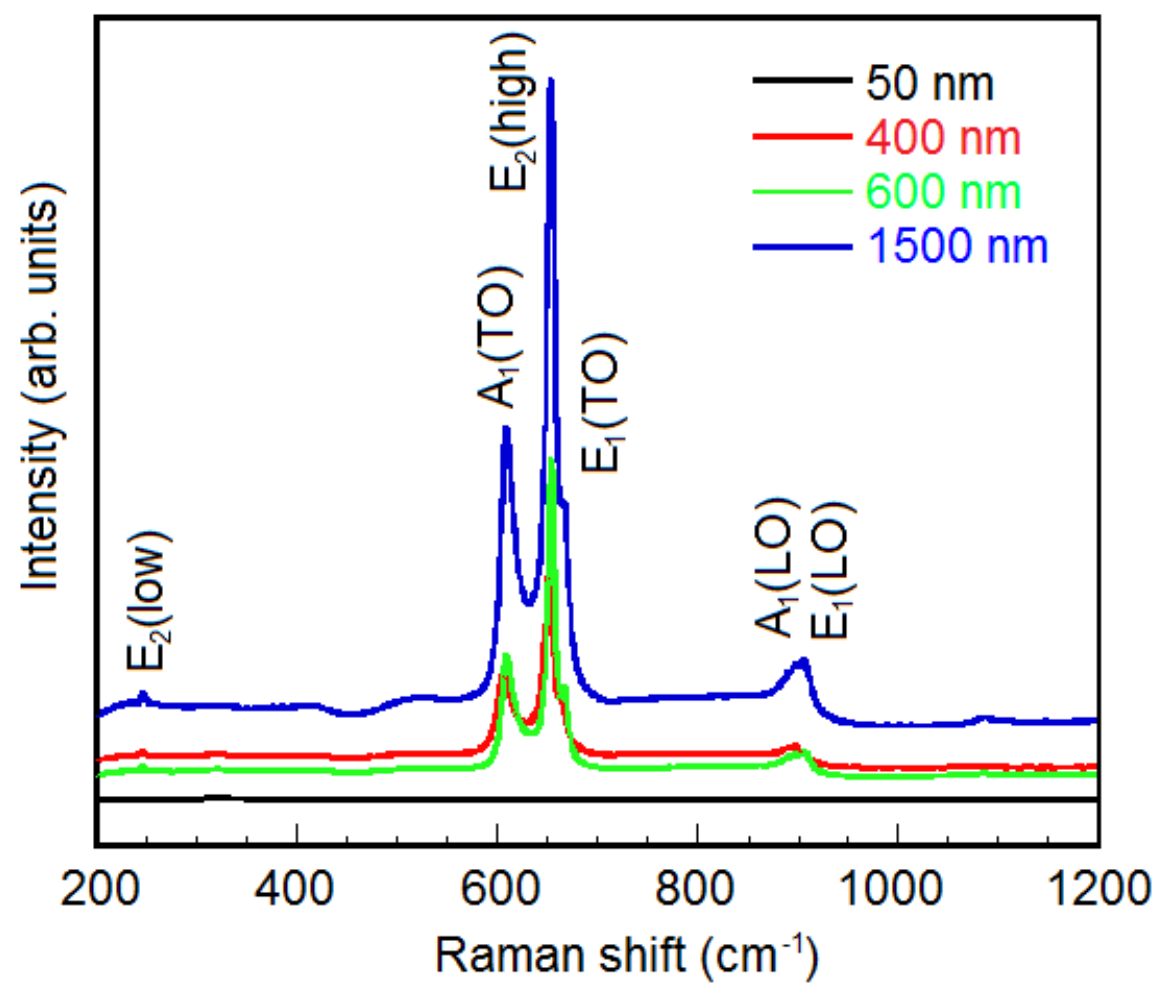

Fig. 4 


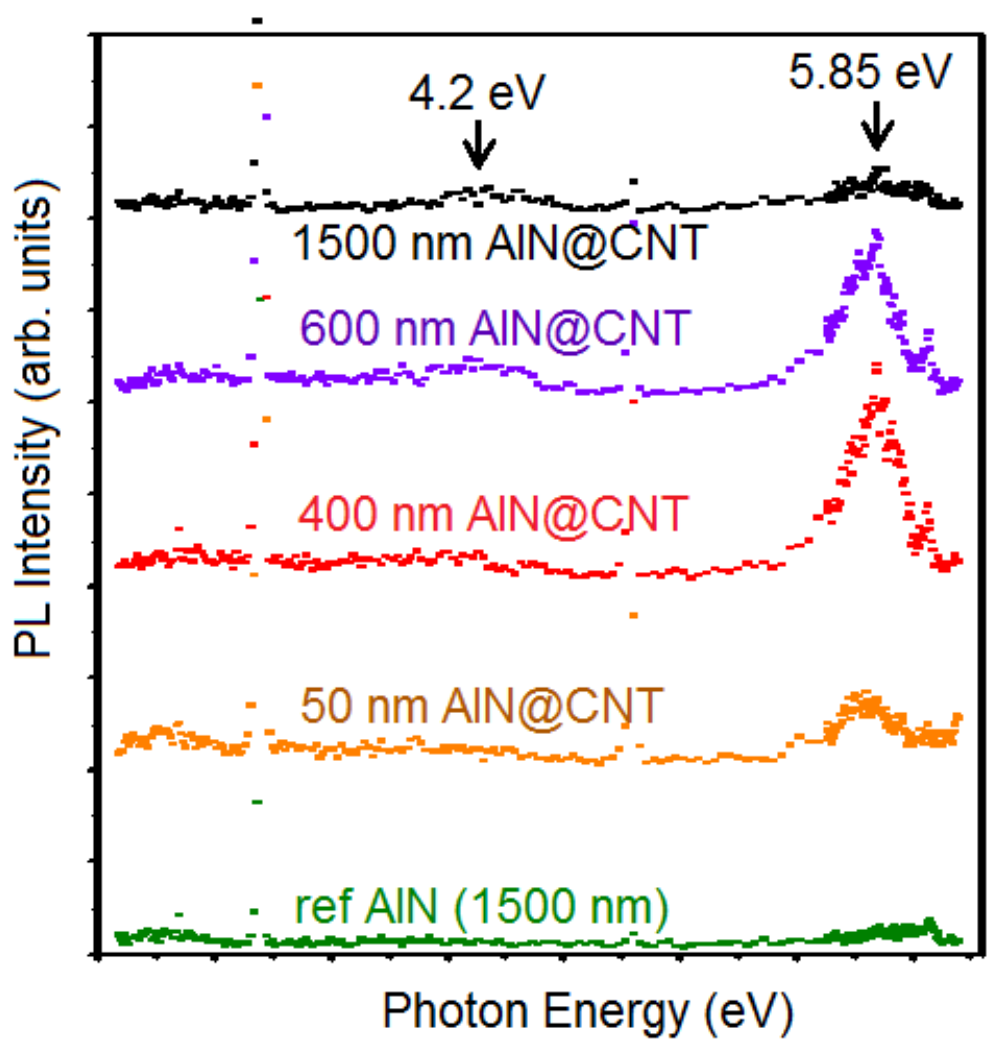

Fig. 5 


\section{Highlights}

- AlN films with 50 to $1500 \mathrm{~nm}$ thickness were produced over vertically aligned CNTs.

- AlN/CNT shell/core assembly exhibits enhancement in PL properties.

- The $5.85 \mathrm{eV}$ exciton band intensity maximizes for $600 \mathrm{~nm}$ think AlN film over CNTs.

- The AIN/CNT shell/core nanoassembly can be used for deep UV optoelectronic devices. 